\title{
Zehn Jahre Neuroethik des pharmazeutischen kognitiven Enhancements - Aktuelle Probleme und Handlungsrichtlinien für die Praxis
}

\author{
Neuroethics of Pharmaceutical Cognitive Enhancement: The First Ten Years - \\ Current Problems and Practical Guiding Principles
}

Autor

Institut

\section{T. K. Metzinger}

Philosophisches Seminar, Johannes Gutenberg-Universität Mainz

\author{
Schüsselwörter \\ - Neuroethik \\ - pharmazeutisches kognitives \\ Enhancement \\ - Krankheitserfindung \\ - Handlungsautonomie \\ - Psychostimulantien \\ - Forschungspolitik

Key words
neuroethics
pharmaceutical cognitive
enhancement
disease mongering
treatment autonomy
psychostimulants
research politics

Bibliografie

DOI http://dx.doi.org/

10.1055/s-0031-1282051

Fortschr Neurol Psychiat 2012;

80: 36-43 @ Georg Thieme

Verlag KG Stuttgart . New York .

ISSN 0720-4299

Korrespondenzadresse

Prof. Dr.

Thomas Karl Metzinger

Philosophisches Seminar,

Johannes Gutenberg-Universität

Jakob Welder Weg 18

5099 Mainz

metzinger@uni-mainz.de

\section{Zusammenfassung}

$\nabla$

Ein auswertender Überblick über die Entwicklung der Neuroethik des pharmazeutischen kognitiven Enhancements (PCE) innerhalb der letzten zehn Jahre, mit einem Schwerpunkt auf der Situation in der Bundesrepublik Deutschland. Der Artikel benennt die wichtigsten begrifflichen Probleme, aktuelle Substanzen und zentrale ethisch-juristische Fragestellungen; am Ende werden allererste Handlungsrichtlinien und Empfehlungen für die Politikgestaltung formuliert.

\section{Was ist "Neuroethik" und was ist "Cognitive Enhancement"?}

„Neuroethik” ist der Name für eine neue akademische Disziplin, die sich mit den ethischen, anthropologischen und soziokulturellen Fragestellungen auseinandersetzt, welche sich aus dem Erkenntnisfortschritt in den Neurowissenschaften ergeben [1]. Von der wissenschaftstheoretischen Systematik her ist die Neuroethik in der Philosophie angesiedelt, weil es in ihrem Kern um Antworten auf normative Fragen geht. Dabei tauchen allerdings momentan viele der konkreten Probleme für die angewandte Ethik im medizinischen Bereich auf, weil es hier zum Beispiel um die neurotechnologische Umsetzung dieses Erkenntnisfortschritts auf dem Gebiet von Therapie und Diagnose geht. Es hat sich eingebürgert, in wissenschaftshistorischer Hinsicht die Entstehung dieses neuen Forschungsbereichs auf das Jahr 2002 zu datieren und mit einer wissenschaftlichen Konferenz zu identifizieren, die vom 13.14. Mai unter dem Titel Neuroethics: Mapping the Field in San Francisco stattfand [2, 3]. Eine erste Fachzeitschrift existiert („Neuroethics“; ZDB-ID 2421622 - 627), und mittlerweile ist es vor allem durch eine Initiative des BMBF auch in Deutschland gelungen, erste Schritte in Richtung auf eine

\section{Abstract \\ $\nabla$}

An evaluating survey of the development of the neuroethics of pharmaceutical cognitive enhancement (PCE) during the last decade, focussing on the situation in Germany, has been undertaken. This article presents the most important conceptual problems, current substances and central ethical and legal issues. Very first guidelines and recommendations for policy-makers are formulated at the end of the text.

institutionelle Verankerung des neuen akademischen Fachs zu unternehmen.

Unter cognitive enhancement (CE; auch „kognitives Enhancement“ oder „Neuroenhancement“) versteht man generell alle Versuche, die geistige Leistungsfähigkeit von Gesunden zu verbessern bzw. auf ein funktionales Niveau hin zu optimieren, das über dem einer durchschnittlichen gesunden Person liegt. Typischerweise geschieht dies heute durch die Einnahme von in der psychopharmakologischen Forschung neu entwickelten Substanzen (PCE; pharmazeutisches kognitives Enhancement; [4-6, 7-12]). Selbstverständlich gibt es neben den legalen Klassikern Koffein und Nikotin eine große Anzahl von körpereigenen Substanzen, Phytopharmaka, Nahrungszusätzen und schon länger bekannten Neuropsychopharmaka, die alle eine geistige Leistungssteigerung hervorrufen können (vgl. dazu [13], siehe dort Tabelle 1), und es existieren auch erste Versuche zur positiven Beeinflussung kognitiver Zustände durch elektrische Hirnstimulation mit Verfahren wie ECT, tDCS, TNS, DBS, EpCS oder MST [14]. Der Versuch, die eigene geistige Leistungsfähigkeit zu verbessern, ist eine uralte Menschheitstradition und für sich genommen auch eine durchaus positive ethisch-zivilisatorische Wertvorstellung. Trotzdem ist in modernen Wettbe- 
werbsgesellschaften eine neue Qualität entstanden, und zwar nicht nur durch Fortschritte in der Hirnforschung und ein immer besseres Verständnis möglicher Wirkmechanismen, sondern insbesondere auch durch neue kommerzielle Zielsetzungen wie der Optimierung und effizienteren „Bewirtschaftung“ des gesunden menschlichen Geistes. Eine genauere Definition des PCE wäre die als einer Technologie, deren Ziel darin besteht, bei Gesunden eine bestimmte Klasse von informationsverarbeitenden Funktionen zu optimieren, nämlich solche Funktionen, die wir heute noch als kognitive Funktionen bezeichnen und die physikalisch im menschlichen Gehirn realisiert sind. Dabei ist häufig unklar, was noch als Therapie und was schon als Enhancement oder rein „kosmetische Psychopharmakologie“ $[15,16]$ zu gelten hat.

\section{Die beiden wichtigsten begrifflichen Probleme: Was wird verbessert? Was ist eine Verbesserung? $\nabla$}

Es gibt gute Argumente dafür, dass sich manche kognitiven Vorgänge in einem wörtlichen Sinne über das Gehirn der jeweiligen Person hinaus in die Umwelt erstrecken, und zwar, weil die sie konstituierenden Informationsverarbeitungsfunktionen partiell auch auf externen Trägern realisiert sind, etwa Computern, dem Internet oder den Gehirnen anderer Menschen ([4], S. 246; [17, 18]). Aber auch auf der Ebene der Inhalte und spezifischen geistigen Leistungen selbst ist häufig unklar, was genau als „Kognition“ gilt: Logisches Denken, Begriffsbildung, Spracherwerb, Textverstehen, mentale Abstraktion, symbolisches Problemlösen, Planung, episodisches Gedächtnis, Entscheidungsfindung oder Metakognition sind zunächst offensichtliche Beispiele. Menschliche Intelligenz resultiert jedoch zum großen Teil aus unbewussten und subsymbolischen Verarbeitungsmechanismen wie der Sinneswahrnehmung, motorischen Simulationen, Aufmerksamkeitskontrolle, Introspektion oder empathiebasierten Formen der sozialen Kognition. Für eine seriöse ethische Bewertung ist es nun entscheidend, dass die Handlungsziele genau spezifiziert werden, d.h. dass man auch weiß, welche kognitive Funktionen tatsächlich optimiert werden sollen (z. B. Aufmerksamkeit oder Arbeitsgedächtnis), welche Effekte möglicherweise nur auf einem Placeboeffekt beruhen (z. B. erhöhte Erfolgserwartung durch eine subjektiv erlebte Vigilanzsteigerung), und welche anderen Funktionen gegebenenfalls abgeschwächt werden (etwa die Fähigkeit zur Impulskontrolle). Für viele der momentan verfügbaren und PCE-relevanten Substanzen ist dies nicht bekannt.

„Intelligenz“ ist ein komplexes theoretisches Konstrukt, dem eine komplexe menschliche Eigenschaft entspricht, die sich nicht monokausal durch Einzelinterventionen kontrollieren lässt. Da die verschiedenen Wirkungen einer die kognitive Leistung verbessernden Substanz in einem komplexen System wie dem menschlichen Gehirn sich gleichzeitig linear und exponentiell entfalten können ([20], S.77), ist durchaus denkbar, dass eine lokale Optimierung zu einer globalen Verschlechterung führt, weil Funktionen in anderen kognitiven Domänen stärker gehemmt oder moduliert werden als innerhalb des eigentlich avisierten Zielbereichs. Empirisch plausible Möglichkeiten sind hier zum Beispiel die Beeinträchtigung des Arbeitsgedächtnisses durch eine Optimierung des Langzeitgedächtnisses, eine Verringerung der Lernfähigkeit und Anpassung an neue Umweltgegebenheiten durch eine pharmakologische Konsolidierung des Langzeitgedächtnisses oder eine Verminderung der behavioralen Flexibilität als Preis für eine Erhöhung der kognitiven Stabilität, etwa zur besseren Aufrechterhaltung der Inhalte des Arbeitsgedächtnisses ([20], S.78; [8]).
Das zweite Hauptproblem besteht in einer begrifflich klaren Definition von „Enhancement“. Zunächst muss man sehen, dass aus streng logischer Perspektive die Unterscheidung zwischen Therapie und Enhancement weder exklusiv noch erschöpfend ist: Eine bestimmte Form des ärztlichen Handelns könnte gleichzeitig therapeutisch und optimierend sein, und es könnte möglicherweise auch einen illegalen Einsatz leistungssteigernder Substanzen geben, der weder Teil einer ärztlichen Heilbehandlung noch primär optimierend ist. Zum Beispiel könnte PCE bei individuellen Patienten mit vormals extrem überdurchschnittlicher geistiger Leistungsfähigkeit, die altersbedingt auf das statistische Durchschnittsniveau abgesunken sind, durchaus als Therapie konzipiert werden; andererseits zeigen neuere empirische Studien, dass nur 10,9\% bzw. 9,2\% der ADHD-Patienten im Erwachsenenalter eine Therapie mit verschreibungspflichtigen Medikamenten erhalten haben, was die Vermutung nahelegt, dass es sich hier in vielen Fällen faktisch eher um Selbstmedikation als um echtes PCE handelt [21]. Eine klassische bioethische Definition würde sagen, dass es sich bei Enhancement generell um alle Funktionsverbesserungen handelt, deren Zielsetzung jenseits der bloßen Aufrechterhaltung oder Wiederherstellung des gesunden Zustandes liegt ([22], S. 29). Es gibt jedoch auch die These, dass bereits alles, was den Organismus - wie z. B. spezielle Diäten, Impfungen oder Implantate - vor einer spezifischen Vulnerabilität schützt, notwendigerweise als Enhancement gelten muss ([23], S. 57; [24]).

Wenn man den gesunden Zustand als „biologischen Normalzustand" definiert (z. B. als funktionale, spezies-spezifische Größe; dazu [26], S. 92; [25]), dann zeigt sich deutlich, dass es eine statistische und eine normative Lesart des Begriffs „normal“ gibt [27], und dass die Semantik beider Lesarten einem historischen Veränderungsprozess unterworfen ist. Zum Beispiel verändern sich die objektive, statistische Verteilung der Symptome des „altersbedingten kognitiven Leistungsabbaus“ oder auch die allgemeine geistige Leistungsfähigkeit in einer Gesellschaft mit der Verfügbarkeit neuer Medikamente, der Verbesserung des Lebensstandards, neuer medialer Umwelten usw. Es ändern sich auch medizinische Lehrbuchdefinitionen [28] und vor allem die subjektive Wahrnehmung dessen, was von den Mitgliedern einer Gesellschaft als „ein Zustand des vollständigen körperlichen, geistigen und sozialen Wohlergehens“ (WHO-Definition von „Gesundheit") erlebt wird. Mit dem soziokulturellen Kontext verschieben sich wiederum die moralischen Intuitionen der Beteiligten und damit auch die Semantik von "normal“ im ethischen Sinne: Ist es wirklich notwendig, dass Lernfähigkeit, Gedächtnis und die Aufmerksamkeitsspanne ab 55 stärker nachlassen? Ist es ethisch geboten, sich neurodegenerativen Vorgängen passiv auszuliefern, oder handelt es sich dabei eigentlich schon heute eher um eine Form von Ungepflegtheit oder Verwahrlosung? Die dynamische Interaktion zwischen „Normalität“ (im deskriptiven Sinne) und „Normalisierung“ (im normativen Sinne) ist komplex, und die begriffliche Unterscheidung zwischen Therapie und Enhancement kann deshalb selbst ein normativer Akt sein ([4], S. 24 ff.).

Der klassische „naturalistische Fehlschluss“ besteht dabei in der falschen Annahme, dass es eine Implikationsbeziehung geben könnte, die von bestimmten deskriptiven Aussagen zu normativen Konklusionen führt ([29], [30], Kapitel 9). In der neuroethischen Bewertung einzelner Substanzen taucht dieser Fehlschluss in der Annahme auf, „natürliche“ Psychopharmaka seien in irgendeinem Sinne wertvoller oder risikoärmer als synthetische Substanzen [31] oder in der Idee, dass „künstliche“ Substanzen im Falle des emotionalen PCE durch moderne Stimmungsaufheller nur zu „unechten“ Ergebnissen und „unauthentischen“ Gefühlen führen kön- 
nen [32]. Ganz allgemein findet man die moralische Intuition, die zum naturalistischen Fehlschluss führt, häufig in der Vorstellung, „natürliche“ Methoden zur Steigerung der geistigen Leistungsfähigkeit (z. B. Meditation, Sport oder richtige Ernährung) seien in einem prinzipiellen und spezifisch ethischen Sinne direkten Interventionen, etwa mit Hilfe pharmakologischer Instrumente, vorzuziehen.

\section{Die wichtigsten aktuellen Substanzen \\ $\nabla$}

PCE erfolgt heute überwiegend durch den nicht bestimmungsgemäßen oder zulassungsüberschreitenden Gebrauch (off-label use) der folgenden drei Substanzklassen:

- Psychostimulantien (insbesondere Methylphenidat und Modafinil),

- Antidementiva (Piracetam, Antiracetam, Pyritional; Memantin, neuere Acetylcholinesterasehemmer wie Rivastigmin oder Donezepil), sowie

- Antidepressiva (SSRIs).

Methylphenidat ist ein etabliertes Instrument in der ADHS-Therapie [33, 34], wird aber besonders in den USA häufig illegal von Studenten eingenommen $[35,36]$. Methylphenidat hemmt im Wesentlichen die Wiederaufnahme von Dopamin und Noradrenalin und erhöht so deren Konzentration im synaptischen Spalt, was unter anderem zu einer Erhöhung des Sympathikotonus führt. Außerdem löst Methylphenidat (in geringerem Ausmaß) eine Freisetzung von Katecholaminen aus und wirkt zudem als Agonist am Serotonin-Rezeptor 5-HT1A und 5-HT2B. 1944 erstmals synthetisiert und als möglicher Nachfolger von Amphetamin konzipiert, wurde Methylphenidat 1954 erstmals auf dem deutschsprachigen Markt eingeführt. Die Substanz hat einen geringen positiven Effekt auf die Gedächtnisleistung und bei einigen Probanden eine subjektiv stimulierende Wirkung, wogegen bei Gesunden konsistente, objektive Effekte auf Stimmung, Aufmerksamkeit und Wachheit nicht belegt sind $[37,38]$. Ein neuerer Trend in der ADHS-Forschung ist die Ergänzung der etablierten, rein symptomatischen Pharmakotherapie mit Psychostimulantien durch Substanzen mit anderen Wirkmechanismen $[39,40]$.

Das pädiatrische Neuroenhancement durch Ritalin wirft neben der besonderen Bedeutung von Sicherheitsaspekten und der Unmöglichkeit eines informed consent weitere spezifische Probleme für die Neuroethik des PCE auf: Wie verändert die Medikation die Persönlichkeitsentwicklung und die Stabilität der Selbstwahrnehmung bei Kindern? Neuere Studien zeigen, dass das bewusste Erleben der eigenen transtemporalen Identität relativ robust zu sein scheint: Amerikanische Kinder betonen interessanterweise ihre bessere schulische Leistungsfähigkeit; britische Kinder die Verbesserung ihrer sozialen Verträglichkeit [ 41 - 43]. Soziale Normen verändern die Selbstbeschreibung, aber nicht primär die moralische Persönlichkeit des Kindes oder die Selbstwahrnehmung als moralischer Agent. In bestimmten sozialen Nischen werden Kinder demnach leistungsoptimierende Neurotechnologien leichter akzeptieren, weil sie einer individualistischen Leistungsethik eher entsprechen und diese moralische Verpflichtung auch schnell „naturalisieren“, d. h. als objektive Gegebenheit akzeptieren. Sind Kinder prinzipiell vulnerabel dafür, bestimmte Aspekte ihrer Persönlichkeitsstruktur zu opfern, weil die soziale Ökologie ihrer moralischen Nische das verlangt? Erste empirische Studien scheinen zu zeigen, dass die moralisch-agentivische Persönlichkeit bei Kindern sehr robust ist und nicht leicht durch eine Behandlung mit Ritalin manipuliert wird, wobei jedoch die Vulnerabilität für soziale Einflüsse beim Einsatz einer solchen neuen Technologie unterschiedlich ist.

Unter den Psychostimulantien mit nicht amphetaminartiger Struktur ist besonders Modafinil hervorzuheben. Der Wirkmechanismus der Substanz ist komplex und noch nicht vollständig verstanden (vgl. [44], sieht dort Tabelle 1). In Deutschland wurde Modafinil im März 2008 aus den betäubungsmittelrechtlichen Vorschriften entlassen und in die normale Verschreibungspflicht überführt, da sein Suchtpotenzial als gering eingeschätzt wird. Momentan ist die Substanz zur Behandlung von Erwachsenen mit exzessiver Schläfrigkeit zugelassen, die mit Narkolepsie, mit und ohne Kataplexie, einhergeht. Modafinil ist aktuell Gegenstand intensiver Forschungsaktivitäten und hat bei Schlafentzug nicht nur positive Effekte auf Wachheit, sondern auch auf Exekutivfunktionen und die Gedächtnisleistung; bei längerem Schlafentzug bleibt nur der Wachheitseffekt erhalten. Nebenwirkungen sind selten und moderat, ohne Schlafentzug sind die beobachteten Wirkungen bei Gesunden inkonsistent und eher schwach, in einigen Studien wurde eine Tendenz zur Überschätzung der eigenen kognitiven Leistungsfähigkeit festgestellt [38, 44]. International steigen die Verkaufsraten ständig an.

Bei den Antidementiva kann man zwischen älteren Substanzen wie Piracetam, Antiracetam, Pyritinol und neueren Acetylcholinesterasehemmern wie Rivastigmin, Galantamin, Donezepil oder Antagonisten des NMDA-Glutamatrezeptors wie etwa Memantin unterscheiden. Donezepil erzielt seine Wirkung über die reversible Blockade des aktiven Zentrums des Enzyms Acetylcholinesterase, wodurch die hydrolytische Spaltung des Neurotransmitters Acetylcholin in Acetat und Cholin verlangsamt wird. Memantin, das in den USA zur Therapie der Alzheimerdemenz zugelassen ist, greift am glutamatergen System als nicht kompetitiver Antagonist des NMDA-Rezeptors an und besitzt eine signifikante, aber geringe therapeutische Wirksamkeit. Für Donezepil und Memantin lässt sich eine positive Wirkung im Sinne eines erfolgreichen PCE bei Gesunden nicht verlässlich nachweisen; generell reicht die Datenlage derzeit nicht aus, um belastbare Aussagen über das Potenzial zur Verbesserung der geistigen Leistungsfähigkeit bei nicht an Demenz erkrankten Personen treffen zu können [45]. Auch bei den Antidepressiva lassen sich momentan für Gesunde keine konsistenten empirischen Belege für eine positive Beeinflussung kognitiver Funktionen und auch nur sehr schwache Hinweise auf eine stimmungsaufhellende Wirkung nach wiederholter Verabreichung angeben [46], obwohl gerade der Glaube an die zuletzt genannte Wirkung weit verbreitet ist [15, 47]. Zusammenfassend lässt sich festhalten, dass der nicht medizinische Gebrauch der genannten Substanzklassen gegenwärtig in Deutschland bei weitem nicht so ausgeprägt ist wie in den USA oder Großbritannien ([6], S.199; [39, 48-53]), und dass die Belege für eine substanzielle und nachhaltige Verbesserung der kognitiven Leistungsfähigkeit durch solche Stoffe ganz im Gegensatz zur öffentlichen, medial vermittelten Wahrnehmung eher schwach sind ([50], siehe dort Tabelle 1). Durchaus plausibel erscheint jedoch die Annahme, dass in der Zukunft wesentliche wissenschaftliche Fortschritte (z.B. auf den Gebieten der Pharmakogenomik, der molekularen Psychiatrie oder der Alzheimerforschung) zu erwarten sind und dass damit auch die Nachfrage nach einem PCE für Gesunde ansteigen könnte. Aus diesem Grund bleiben nicht nur die objektiven Probleme einer rationalen Nutzen-Risiko-Abwägung weiter bestehen, sondern insbesondere auch die ethischen und juristischen Fragestellungen [54]. 


\section{Die wichtigsten ethischen und juristischen Fragestellungen: Zentrale Punkte der aktuellen normativen Debatte \\ $\nabla$}

Eingangs wurde bereits darauf hingewiesen, dass die Neuroethik systematisch in der Philosophie angesiedelt ist, und zwar nicht nur, weil die Ethik eine Teildisziplin der Philosophie ist, sondern weil es in ihrem Kern um die Möglichkeit von Antworten auf normative Fragen geht. Eine hilfreiche begriffliche Unterscheidung ist hier die zwischen „deskriptiver Neuroethik“ und „normativer Neuroethik“, die sich jeweils durch ganz unterschiedliche Erkenntnisziele und Fragestellungen auszeichnen. Hier sind einige typische Beispiele:

- Deskriptive Neuroethik

- Was sind die objektiven neuronalen Grundlagen moralischer Emotionen? Wie lassen sich rein empirisch die notwendigen und hinreichenden Bedingungen isolieren, durch die Empathiefähigkeit, erfolgreiche Perspektivenübernahme und soziale Kognition konstituiert werden?

- Was ist die evolutionäre Geschichte menschlichen Moralverhaltens, des Altruismus, der moralischen Kognition und der Fähigkeit zu ethischer Einsicht?

- Was sind auf molekularer Ebene die genauen Wirkmechanismen einer bestimmten pharmakologischen Substanz? Was ist ihr objektives Nutzen-Risikoprofil bei Gesunden?

- Normative Neuroethik

- Wenn man mit vertretbarem Risiko die ethische Einsichtsfähigkeit und das prosoziale, altruistische Verhalten bei Gesunden verbessern könnte, entstünde dann eine moralische Verpflichtung, dies auch tatsächlich zu tun („moral enhancement“; [4], S. 257 f., [55 - 58])? Sollten wir in forschungspolitischer Hinsicht diesen Bereich besonders fördern?

- Angenommen, wir entscheiden uns für eine teilweise und kontrollierte Freigabe des PCE bei Gesunden: Was genau bedeuten dann staatliche Fürsorgepflicht, Chancengleichheit und Verteilungsgerechtigkeit?

- Sollte eine militärische Anwendung pharmazeutischer Neurotechnologien generell verhindert werden, oder gibt es Anwendungsbereiche, in denen sie sogar ethisch geboten ist?

Untersucht man die neuroethische Fachliteratur seit 1985 (vgl. http://www.neuroethik.ifzn.uni-mainz.de/), dann stellt man interessanterweise fest, dass die Anzahl von Publikationen zur normativen Neuroethik - im Gegensatz zu primär deskriptiv operierenden, empirischen Studien - sowie von Forschungsbeiträgen genuin philosophischer Autoren eher gering ist. Was die genaue Erklärung für dieses Phänomen sein könnte, ist gegenwärtig noch unklar. Ein Desiderat für die zukünftige Forschungsförderung besteht jedoch darin, diese Unausgewogenheit mittelfristig zu beseitigen. Im Folgenden werde ich deshalb kurz einige der relevantesten normativ-philosophischen Probleme skizzieren.

\section{Schwierigkeiten der terminologischen Trennung von normativen und nicht-normativen Bedeutungselementen}

Matthis Synofzik hat darauf hingewiesen, dass Begriffe wie „Krankheit“, „Normalität“ oder auch „Medizin“ häufig einen rein rhetorischen und „kryptonormativen“ ([26], S. 89) Charakter besitzen, weil sie implizite, aber ethisch ungerechtfertigte Implikationen mit sich bringen. Sie können deshalb die argumentative Last einer echten Begründung nicht tragen, etwa als logische Abgrenzungskriterien in der klinisch-praktischen Anwendung. Dies gilt dann natürlich auch für klassische bioethische Prinzipien wie das Nichtschädigungsgebot (Non-Malefizienz), das Gebot zur Hilfe (Benefizienz), das Gerechtigkeitsprinzip und die wichtigen Ideen der Patientenautonomie oder auch der partizipativen Arzt-Patienten-Beziehung, bei der der medizinische Experte gewissermaßen als Mentor in einem kooperativen Entscheidungsfindungsprozess fungiert. Diese Prinzipien sind zwar wesentlich kontextsensitiver, leichter anwendbar und für die medizinische Praxis geeigneter als abstrakte ethische Konzepte. Trotzdem bleibt die Frage bestehen, in welchem Sinne sie als ethisch gerechtfertigt gelten können, weil sie auf einer echten, genuin normativen Art von Wissen basieren. Können normative Aussagen überhaupt wahr oder falsch sein (und damit eine eigene Form von Erkenntnis ausdrücken), und zwar, weil es objektive, normative Tatsachen („moralische Fakten“) gibt, auf die solche Sätze sich beziehen können? Oder gibt es so etwas wie eine Erkenntnis ethischer Tatsachen in einem strengen Sinne nicht? Dies ist vielleicht der eigentliche Kern der philosophischen Problematik.

In jedem Fall ist es für die neuroethische Bewertung des PCE von großer Bedeutung, die rein deskriptiven und empirisch überprüfbaren (also prinzipiell erkennbaren) Komponenten eines Problems sauber von den normativen (möglicherweise nicht konsensfähigen) Bestandteilen zu trennen. Zum Beispiel zeigt eine genauere wissenschaftstheoretische Untersuchung medizinischer Klassifikationssysteme eine Interaktion von epistemischen und nicht-epistemischen Aspekten, da die zur Systematisierung von diagnostischen Entitäten eingesetzten Validatoren selbst eine inhärente Normativität besitzen, die methodologisch nicht zu eliminieren ist [28]. „Validatoren“ sind dabei Kriterien, die etwa im ICD und im DSM eingesetzt werden, um bestimmte Krankheiten genauer zu klassifizieren und zu systematisieren, zum Beispiel, um eine Alzheimerdemenz von einer anderen Form von Demenzerkrankung abzugrenzen oder von einer nichtpathologischen Form des „kognitiven Alterns“. Manche (,nichtepistemischen“) Aspekte solcher Kriterien lassen sich nicht vollständig verobjektivieren und stellen deshalb selbst Wertentscheidungen dar, die jedoch oft nicht als solche erkannt werden. Dasselbe gilt aber zum Beispiel auch für den Ausschluss nichtkognitiver Aspekte - wie dem vermehrten Auftreten von Angstoder Erregungszuständen - aus den diagnostischen Kriterien für die Alzheimerdemenz: Es handelt sich nicht nur um eine pragmatische, sondern eben auch um eine wertende (also normative) Entscheidung, die dazu führen kann, dass sehr frühe Formen des kognitiven Leistungsabbaus (die möglicherweise im traditionellen Verständnis noch nicht pathologisch sind) nun behandelt werden, während Veränderungen im Verhalten oder emotionalen Selbsterleben des Patienten in der therapeutischen Praxis ignoriert werden, obwohl sie möglicherweise mit einem deutlich stärkeren subjektiven Leidensdruck einhergehen.

\section{Marktinteressen und die Konstruktion neuer diagnostischer Entitäten}

Ein zweites wichtiges Stichwort innerhalb der aktuellen Debatte ist disease mongering [59, 60]. Bei diesem Vorgang der interessengeleiteten Erfindung von Krankheiten geht es darum, neue diagnostische Entitäten zu konstruieren, um neue Märkte für all jene zu entwickeln, die Medikamente und Heilmethoden verkaufen. In den Worten von Ray Moynihan et al.: „It is a process that turns healthy people into patients, causes iatrogenic harm, and wastes precious resources. Disease mongering is the contemporary form of 'medicalization'” ([59], e106; [20], S. 81). Seit dem Erscheinen der Erstausgabe des statistischen Handbuchs der 
American Psychiatric Association im Jahre 1952 sind mehr als vierhundert neue Kategorien von geistigen Erkrankungen gebildet worden [61]. Natürlich sind diese neuen Kategorisierungen in klinischen Beobachtungen und theoretischen Erwägungen verankert und nehmen auf vorfindliche und empirisch ausweisbare Phänomene Bezug. Im Gegensatz zum ganz normalen Vorgang der Konstruktion neuer semantischer Entitäten in der Theorieentwicklung der meisten anderen naturwissenschaftlichen Fächer steht die psychiatrische Nosologie dabei aber unter einem wesentlich stärkeren Druck durch die Industrielobby und weltanschauliche pressure groups. Für die Neuroethik des PCE ist dieser Zusammenhang besonders wichtig, weil gerade hier große Zukunftsmärkte und massive finanzielle Interessen im Entstehen begriffen sind.

\section{Authentizität, personale Identität und Selbstbestimmung}

Viele aktuelle Debatten um den möglichen Verlust von „Authentizität“ bei Erwachsenen gehen von einem begrifflich unklaren und empirisch unplausiblen Begriff des Selbst als einer mysteriösen und unveränderlichen Essenz aus, einem eindeutig bestimmbaren, echten oder „wahren“ Kern der Person [62 - 65]. Ein verwandtes Problem ist die immer wieder auftretende Frage nach dem möglichen Verlust der „personalen Identität“ [66]. Dabei ist fast nie das philosophisch-ontologische Problem der numerischen oder transtemporalen Identität von Personen gemeint (auf das dieser etablierte Fachterminus sich in Wirklichkeit bezieht; $[67,68])$, sondern das Risiko einer inakzeptablen Veränderung von Persönlichkeitsmerkmalen im psychologischen Sinne ([69]; [72], S. 189 ff.). Persönlichkeitsmerkmale in einem operationalisierten Sinn sind relativ zeitstabile Mengen von Verhaltensdispositionen. Häufig wird einerseits übersehen, dass Persönlichkeitsmerkmale generell relativ stabil sind (vgl. dazu auch die zitierten Studien zum pädiatrischen Neuroenhancement mit Ritalin), dass aber andererseits bereits das normale Leben - oder auch der typische Verlauf einer nicht ausreichend behandelten Erkrankung - Veränderungen im Profil der Charaktermerkmale einer Person mit sich bringen können. Jan Bublitz und Reinhard Merkel haben überzeugend argumentiert, dass der eigentliche Kern des Problems in der Beziehung zwischen dieser Tatsache und der Frage nach der Autonomie einer Person besteht: Entscheidend ist, ob sich unter dem Einfluss von PCE die moralische Einsichtsfähigkeit, sowie ein zu definierendes Mindestmaß von Rationalität und Selbstkontrolle erhalten, und ob sich die Person auf psychologisch-phänomenologischer Ebene tatsächlich mit ihren aus freier Entscheidung heraus optimierten Persönlichkeitsmerkmalen identifizieren kann [73]. Der diffuse Begriff der „Authentizität“" hilft bei der Lösung dieses Problems nicht weiter. Auf empirischer Ebene gibt es, so Bublitz und Merkel, keinerlei Anzeichen dafür, dass die aktuell verfügbaren Substanzen die Autonomie der Person bedrohen. Man kann in diesem Zusammenhang sogar darauf hinweisen, dass sich ein wichtiger Aspekt der aktuellen Problematik genau daraus ergibt, dass sich der Raum der Handlungsoptionen für den einzelnen Bürger stetig vergrößert.

\section{Die Abwägung zwischen individueller Autonomie und öffentlichen Gütern}

Historisch neu an der aktuellen Situation ist nämlich auch, dass nationale Gesetzgeber die entsprechenden Märkte durch das Aufkommen von Internetapotheken kaum noch wirksam kontrollieren können. Eine neuere Studie fand 157 Internetseiten, auf denen verschreibungspflichtige Medikamente zum Kauf angeboten wurden; keine dieser Internetapotheken besaß einen Kinderschutzmechanismus, nur 6\% forderten Rezepte per Fax oder per Post an [74]. Über Verunreinigungen oder den vorsätzlichen Betrug von Kunden liegen keine gesicherten Daten vor, von einer Dunkelziffer ist jedoch auszugehen. Es ist somit auch ein neuer Handlungsraum entstanden, der nicht nur von unseriösen Anbietern, sondern auch von potenziellen Konsumenten durchaus als rechtsfrei wahrgenommen werden kann. Zwar wissen wir aus der Vergangenheit, dass sich das Konsumentenverhalten effektiver durch kulturelle Kontexte kontrollieren lässt als durch Gesetze - was ja gerade die Bedeutung rationaler, öffentlicher Debatten unterstreicht, die im Idealfall solche neuen Kontexte erzeugen können. Die klassische Frage im Hintergrund ist jedoch, wie weit in offenen, demokratischen Gesellschaften die Freiheit des Individuums maximiert werden kann, ohne die Interessen der Allgemeinheit dauerhaft zu beschädigen.

Der in diesem Zusammenhang eingeführte Begriff der „cognitive liberty“ [75] fußt auf zwei grundlegenden Prinzipien, die die Handlungsautonomie des einzelnen Bürgers schützen und maximieren sollen: Solange das Verhalten des Einzelnen die Interessen anderer nicht verletzt, sollte er - erstens - niemals gezwungen werden können, dem Einsatz von Neurotechnologien (ob pharmakologischer Natur oder nicht) zuzustimmen, welche direkt mit seinem eigenen Gehirn interagieren, und das Individuum darf - zweitens - auch nicht durch Kriminalisierung oder auf andere Weise daran gehindert werden, solche neuen Technologien für sich selbst einzusetzen ([75], S. 227). In der ethisch fundierten Politikberatung muss es jedoch auch um öffentliche Güter gehen und nicht ausschließlich um die Maximierung individueller Autonomie [76]. Saskia Nagel hat zudem herausgearbeitet, dass bestimmte Einschränkungen nicht nur im Interesse des autonomen Individuums selbst liegen, sondern dass die Anerkennung von Autonomie auch in der Anerkennung eines Rechts auf staatlichen Schutz und Begleitung bestehen kann ([77], S.117, [78]). Ein klassisches Beispiel wären hier kulturell etablierte Formen des Enhancements (etwa durch Nikotin), welche bei geringer Wirksamkeit typischerweise auf dem Weg über eine für Konsumenten überraschend schnell eintretende Abhängigkeit zu einem ausgeprägten und nachhaltigen Autonomieverlust führen. Das Recht zur Selbstbestimmung ist nicht dasselbe wie die Kompetenz zur Selbstbestimmung, und ein erweiterter Raum von Handlungsoptionen erhöht immer auch das Risiko von Fehlentscheidungen, wobei auch die Angst vor Reue, gestiegener Selbstverantwortlichkeit und die mentale Repräsentation angewachsener Opportunitätskosten zu neuen Stressoren werden, die den erwarteten Zuwachs an Wohlbefinden und Lebenszufriedenheit wieder minimieren. Es geht hier also nicht nur um rein normative Fragen, sondern um praktische Klugheit und ein empirisch realistisches Menschenbild: Wie widerstandsfähig ist die menschliche Fähigkeit zur Selbstbestimmung tatsächlich angesichts einer potenziell großen Menge neuer Handlungsmöglichkeiten, die ganz direkt die Grundlagen des Ichgefühls und der Handlungskontrolle selbst betreffen? Nagel legt nahe, dass eine sanfte und nicht-paternalistische Unterstützung durch strukturierte Systeme von Wahlmöglichkeiten und Feedbackmechanismen dem einzelnen Bürger und seiner Autonomie letztlich mehr dienen kann als eine undifferenzierte Liberalisierung. 


\section{Geistige Leistungssteigerung bei Gesunden als das ethische Grundproblem}

Die normative Grundfrage für die angewandte Ethik des PCE besteht vielleicht darin, ob es irgendwelche prinzipiellen Einwände gegen eine kognitive Selbstoptimierung unter Einsatz moderner neurotechnologischer Methoden geben kann. Eine pauschale Ablehnung scheint unhaltbar, allein schon deshalb, weil es sich bei der Verbesserung des eigenen Geistes um ein klassisches abendländisches Ideal handelt, das von der Antike bis zur Aufklärung immer wieder neu formuliert worden ist, und natürlich auch weil eine so fundamentale Einschränkung des menschlichen Selbstbestimmungsrechts sich mit den Grundannahmen des westlichen Demokratieverständnisses nicht in Einklang bringen ließe. Auf der anderen Seite ist sicher die Frage berechtigt, ob die Verabreichung leistungssteigernder Medikamente an Gesunde im konkreten Fall überhaupt mit dem ärztlichen Ethos und den ärztlichen Pflichten vereinbar ist.

Diese Grundproblematik zeigt sich deutlich an einer aktuellen Fragestellung, die gegenwärtig kontrovers diskutiert wird und hier zum Abschluss kurz skizziert sei: Sollten wissenschaftliche Langzeitstudien an Gesunden durchgeführt werden, um genauere Daten über Nebenwirkungen und Risiken einer solchen Anwendung zu erheben? Die Gegner solcher Studien weisen zu Recht darauf hin, dass man mit solchen Forschungsprojekten letztlich das Geschäft der Pharmaindustrie betreiben könnte (die ein Interesse an der Erweiterung von Indikationen und der Erschließung neuer Absatzmärkte hat), und dass die Verabreichung von Medikamenten an Gesunde nicht nur dem ärztlichen Ethos widerspricht, sondern schlicht verboten ist. Auf der anderen Seite fordern führende Neuroethiker einen strikt evidenzbasierten Ansatz [6]. In Abwesenheit belastbarer Daten über das tatsächliche Verhältnis zwischen Nutzen und Risiken des PCE bei Gesunden ist es nämlich schwer, eine seriöse ethische Entscheidung zu treffen, die gleichzeitig rational und empirisch fundiert ist. Wenn eines der politischen Ziele der Gesetzgebung in diesem Bereich nun aber die Minimierung individuellen menschlichen Leidens und der allgemeinen psychosozialen Kosten der Gesamtentwicklung ist, und wenn zusätzlich gezeigt werden könnte, dass sich der zulassungsüberschreitende, illegale und unkontrollierte Gebrauch der fraglichen Substanzen bereits auszubreiten beginnt, dann kann man dafür argumentieren, dass es einerseits unethisch und andererseits politisch unklug wäre, die Erhebung solcher Daten zu behindern. Es ist abzusehen, dass dieses Dilemma die Neuroethik in der Zukunft in verschiedenen Varianten immer wieder beschäftigen könnte.

\section{Empfehlungen und erste Handlungsrichtlinien}

Die folgenden Empfehlungen ergeben sich aus der Fachdebatte des letzten Jahrzehnts. Sie beziehen sich ausschließlich auf die Situation in der Bundesrepublik Deutschland, spiegeln lediglich die Meinung des Autors wider und erheben keinen Anspruch auf Vollständigkeit.

\section{Forschungspolitik}

1. In der Bundesrepublik Deutschland ist innerhalb der Disziplin der Neuroethik das Verhältnis von genuin analytisch-ethischer und rein empirisch-datenerhebender Forschung - und somit auch zwischen "normativer" und „deskriptiver“ Neuroethik noch unausgewogen (vgl. S. 39). Der relative Anteil exzellenter analytisch-ethischer Forschungsarbeiten muss erhöht werden.
2. Die generelle ethische Problematik von Langzeituntersuchungen an Gesunden muss insbesondere aus philosophischer Perspektive genauer untersucht werden.

3. Spätestens sobald sich der unkontrollierte zulassungsüberschreitende Gebrauch einer speziellen Substanz faktisch in der Bundesrepublik auszubreiten beginnt, sollten im Sinne der Fürsorgepflicht des Staates ökologisch valide Langzeitstudien aufgelegt werden, die eine rationale Nutzen-Risiko-Abschätzung ermöglichen.

4. Es müssen bessere Instrumente zum Screening, zur Überwachung und zur Evaluation des klinischen Kurzzeitenhancements entwickelt werden ([41], S.13). Substanzen, die auf spezifische Aspekte der kognitiven Leistungsfähigkeit bei Erwachsenen abzielen, sollten parallel immer auch mit Blick auf ihr pädiatrisches Nutzen-Risiko-Profil bewertet werden.

5. Ist die Freigabe einzelner Substanzen geplant, so müssen Instrumente zum Langzeitscreening, zur Überwachung und zur Evaluation der Veränderung und der Stabilität von Persönlichkeitsmerkmalen sowie der individuellen Autonomie (d. h. moralischer Einsichtsfähigkeit, minimaler Rationalität und behavioraler Selbstkontrolle) für das PCE bei Gesunden entwickelt werden.

6. Das neue Fach „Neuroethik“ sollte möglichst bald auch in der universitären Lehre etabliert werden, indem insbesondere für die Fächer Philosophie, Neurowissenschaften und Medizin systematische Curricula und Lehrmaterialien entwickelt werden, die den jeweiligen fachdidaktischen Ansprüchen genügen.

\section{Öffentlichkeitsarbeit}

1. In den ersten zehn Jahren der neuroethischen Debatte hat sich gezeigt, dass viele öffentliche Medien ein Interesse an der systematischen Sensationalisierung der Problematik haben, indem sie sowohl die Risiken, als auch die Wirksamkeit und die tatsächliche gesellschaftliche Verbreitung von PCE übertreiben und skandalisieren. Dies schadet der Entwicklung einer ethisch stimmigen und empirisch angemessenen gesellschaftlichen Antwort, und es schafft Anreize zur unbegründeten Einnahme der fraglichen Substanzen. Die beteiligten Forscher und Institutionen müssen deshalb wesentlich stärker als bisher auf eine sachliche Berichterstattung drängen. Die Rolle und das spezifische Eigeninteresse der Medien als Plattform für notwendig gewordene öffentliche Debatten, aber auch bei der Erzeugung von Nachfrage und Missbrauchsverhalten muss wesentlich kritischer und sorgfältiger analysiert werden als in der Vergangenheit.

2. Die allgemeine Öffentlichkeit hat ein Recht auf kontinuierliche und objektive Information über Wirkungen, Nebenwirkungen, Nutzen und Risiken des PCE. Dies ist auch Aufgabe des Staates. Forschung und Politik müssen deshalb eigene, unabhängige Strategien zur objektiven Information der Öffentlichkeit über Effekte, Nebenwirkungen, Chancen und Risiken in Zusammenhang mit neuen, die kognitive Leistungsfähigkeit steigernden Substanzen entwickeln.

\section{Politikgestaltung}

1. Generell sollte die Politikgestaltung im Bereich der Neurotechnologie streng evidenzbasiert erfolgen und sich von rein ideologischen Debatten fernhalten. Das bedeutet, dass politische Entscheidungen in einer säkularen Demokratie des 21.Jahrhunderts nach Möglichkeit auf rationalen, philosophisch-neuroethischen Argumenten und den verfügbaren empirischen 
Daten beruhen sollten und deshalb strikt von bloß weltanschaulichen Debatten getrennt werden müssen.

2. Finanzielle Anreize zur Verschreibung von leistungssteigernden Medikamenten für die zulassungsüberschreitende Anwendung im Sinne des PCE bei Gesunden sollten auf allen Ebenen beseitigt werden.

3. Der Prozess der Einführung neuer diagnostischer Entitäten muss stärker als in der Vergangenheit kritisch begleitet werden. Die Konstruktion ethisch zweifelhafter, begrifflich unklarer oder nicht eindeutig evidenzbasierter Krankheitskategorien muss eingedämmt werden, weil sie dem Nichtschädigungsgebot widerspricht, Ressourcen verschwendet und neue Risiken erzeugt.

4. Um die Entwicklung auch auf finanz- und gesundheitspolitischer Ebene genauer einschätzen zu können, müssen Daten über die tatsächliche Entwicklung der Kosten in diesem Bereich erhoben werden.

5. Es scheint mittlerweile klar, dass die PCE-Problematik und ihre angemessene politisch-juristisch-ethische Regulierung in den kommenden Jahrzehnten eine vielleicht langsam, jedoch stetig wachsende Rolle spielen werden. Erstens ist mit der Entwicklung neuer und effektiverer Substanzen zu rechnen, zweitens stehen hinter diesem Vorgang erhebliche Marktinteressen, und drittens ist abzusehen, dass durch die demografische Entwicklung sowie die generelle Arbeitsverdichtung und Beschleunigung der Lebenswelt auch das Konsumenteninteresse eher zu- als abnehmen wird.

6. Auf der anderen Seite darf die Politikformulierung selbst dem unkontrollierten Gebrauch neuer Substanzen keinen Vorschub leisten. Insbesondere sollte sie nicht in einer Art vorauseilenden Gehorsam Anpassungen an Zukunftsszenarien vornehmen, die sich möglicherweise in der Bundesrepublik Deutschland so nie realisieren.

7. Wir leben - ob wir es wollen oder nicht - in einer historischen Übergangsphase, die sich immer deutlicher durch Stichworte wie „Naturalistische Wende im Menschenbild“ oder „Technische Kontrollierbarkeit geistiger Eigenschaften“ charakterisieren lässt. Es gibt deshalb einen größeren anthropologischen und soziokulturellen Zusammenhang, in den die hier skizzierte Entwicklung eingebettet ist. Erforderlich sind deshalb auch allgemeinere öffentliche Debatten und vor allem die Erzeugung eines neuen kulturellen Kontextes, der mittelfristig eine rationale und ethisch vertretbare gesamtgesellschaftliche Umsetzung der vielen neuen wissenschaftlichen Erkenntnisse und den aus ihnen resultierenden Handlungsmöglichkeiten gestattet.

\section{Take Home Message}

Die Neuroethik beginnt erfolgreich, sich in der Bundesrepublik Deutschland als eigenständiger und aktiver Forschungsbereich zu etablieren. Auf dem Gebiet der angewandten Ethik des PCE zeigt sich einerseits, dass sich der zulassungsüberschreitende und unkontrollierte Gebrauch der fraglichen Substanzen in Deutschland bei Weitem noch nicht so weit ausgebreitet hat wie etwa in den USA, dass es jedoch andererseits bezüglich des zukünftigen Einsatzes von Stoffen, die die geistige Leistungsfähigkeit von Gesunden erhöhen sollen, auch weiterhin eine ganze Reihe von ethischen, juristischen und politischen Fragen gibt, die einer gründlichen Klärung und stetiger Aufmerksamkeit bedürfen.

\section{Danksagung}

Ich danke drei anonymen Gutachtern der Zeitschrift für ihre detaillierten Verbesserungsvorschläge und ihre konstruktive Kritik, sowie Wanja Wiese und Jennifer M. Windt für technische Hilfe bei der Erstellung des Manuskripts. Die Arbeit an diesem Beitrag wurde durch das Bundesministerium für Bildung und Forschung gefördert, und zwar im Rahmen des deutsch-kanadischen Forschungsprojekts Normality, Normalization and Enhancement in the Neurosciences: Ethical, Sociocultural and Neuropsychiatric Aspects of Cognitive Enhancement (2008-2011).

Interessenkonflikt: Der Autor gibt an, dass kein Interessenkonflikt besteht.

\section{Literatur}

1 Illes J, Sahakian BJ. The Oxford Handbook of Neuroethics. New York: Oxford University Press 2011

2 Marcus SJ (ed.) Neuroethics: Mapping the Field. New York: The Dana Press 2002

3 Farah M. Neuroscience and neuroethics in the 21st centruy. In: Illes J, Sahakian BJ. The Oxford Handbook of Neuroethics New York: Oxford University Press 2011, 761-781

4 Metzinger T, Hildt E. Cognitive enhancement. In: Illes J, Sahakian BJ. The Oxford Handbook of Neuroethics New York: Oxford University Press $2011,245-264$

5 Morein-Zamir S, Sahakian B. Pharmaceutical cognitive enhancement. In: Illes J, Sahakian BJ. The Oxford Handbook of Neuroethics New York: Oxford University Press 2011, 229-244

6 Sahakian B, Morein-Zamir S. Neuorethical issues in cognitive enhancement. J Psychopharm 2011; 25 (2): 197-204

7 Glannon W. Psychopharmacological enhancement. Neuroethics 2008; 1: $45-54$

8 De Jongh R, Bolt I, Schermer $M$ et al. Botox for the brain: enhancement of cognition, mood and pro-social behavior and blunting of unwanted memories. Neurosci Biobehav Rev 2008; 32: 760-776

9 Mehlman MJ. Cognition-enhancing drugs. Milbank Quart 2004; 82 (3): 483-506

10 Larriviere $D$, Williams MA, Rizzo $M$ et al. Responding to requests from adult patients for neuroenhancements: guidance of the Ethics, Law and Humanities Committee. Neurol 2009; 73 (17): 1406 - 1412

11 Lieb K. Hirndoping. Mannheim: Tamtmos, Artemis \& Winkler 2010

12 Schöne-Seifert B, Talbot D (Hrsg) Enhancement. Die ethische Debatte. Paderborn: mentis 2009

13 Förstl H. Neuro-Enhancement - Gehirndoping. Der Nervenarzt 2009; 7: $840-845$

14 Hoy KE, Fitzgerald PB. Brain stimulation in psychiatry and its effects on cognition. Nat Rev Neurol 2010; 6: 267-275

15 Kramer PD. Listening to Prozac. A Psychiatrist Explores Antidepressant Drugs and the Remaking of the Self. Viking Press 1993

16 Cerullo MA. Cosmetic psychopharmacology and the President's Council on Bioethics. Persp Biol Med 2006; 49 (4): 515-523

17 Clark A, Chalmers DJ. Der erweiterte Geist. In: Metzinger T (Hrsg) Grundkurs Philosophie des Geistes. Band III: Intentionalität und mentale Repräsentation

18 Menary R (Hrsg) The Extended Mind. Cambridge: MA: MIT Press 2010

19 Buller T. Neurotechnology, invasiveness and the extended mind. Neuroethics 2011. DOI: 10.1007/s12152-011-9133-9135

20 Schermer M, Bolt I, de Jongh $R$ et al. The future of psychopharmacological enhancements: expectations and policies. Neuroethics 2009; 2: $75-87$

21 Outram SM. Ethical considerations in the framing of the cognitive enhancement debate. Neuroethics 2011. DOI: 10.1007/s12152-0119131-7

22 Jüngst ET. What does enhancement mean? In: Parens E (ed.) Enhancing human traits: ethical and social implications Washington D.C: Georgetown University Press 1998, 29-47

23 Harris J. Enhancing Evolution. Princeton: Princeton University Press 2007 
24 Greely H, Sahakian B, Harris J et al. Towards responsible use of cognitive-enhancing drugs by the healthy. Nature 2008; 456: 702 - 705

25 Daniels $N$. Normal functioning and the treatment-enhancement distinction. Camb Quart Health Eth 2000; 9: 309-322

26 Synofzik $M$. Ethically justified, clinically applicable criteria for physician decision-making in psychopharmacological enhancement. Neuroethics 2009; 2: 89-102

27 Murphy D. Concepts of disease and health. In: Zalta EN (ed.) The Stanford Encyclopedia of Philosophy.(Fall 2009 edition) http://plato.stan ford.edu/archives/sum2009/entries/health-disease/ [Epub only]

28 Kutschenko L. In quest of "good" medical classification systems. Med Stud 2011; 3 (1): $53-70$

29 Birnbacher D. Bioethik zwischen Natur und Interesse. Frankfurt: Suhrkamp 2006

30 Birnbacher D. Analytische Einführung in die Ethik. 2. Aufl. Berlin: de Gruyter 2007

31 Gather J. The evaluation of psychopharmacological enhancers beyond a normative "natural"-“artificial" dichtomoy. Med Stud 2011; 3 (1): $19-27$

32 Krämer F. Authenticity anyone? The enhancement of emotions via neuro-psychopharmacology. Neuroethics 2011; 4: 51-64

33 Singh I. Beyond polemics: science and ethics of ADHD. Nat Rev Neur 2008; 9: 957-964

34 Wilens TE, Adler LA, Adams J et al. Misuse and diversion of stimulants prescribed for ADHD: a systematic review of the literature. J Am Acad Child Adolesc Psychiatry 2008; 47 (1): 21 - 31

35 Low KG, Gendaszek AE. Illicit use of stimulants among college students: A preliminary study. Psych Hea \& Med 2002; 7: 283-287

36 Forlini C, Racine E. Autonomy and coercion in academic "Cognitive Enhancement" using methylphenidate: perspectives of key stakeholders. Neuroethics 2009; 2: 163-177

37 Repantis D, Schlattmann P, Laisney $O$ et al. Modafinil and methylphenidate for neuroenhancement in healthy individuals: A systematic review. Pharmacol Res 2010; 62 (3): 187-210

38 Repantis D. Die Wirkung von Psychopharmaka bei Gesunden. In: Wienke A, Eberbach WH, Kramer HJ, Janke K (Hrsg) Die Verbesserung des Menschen: Tatsächliche und rechtliche Aspekte der wunscherfüllenden Medizin Heidelberg: Springer 2009, 63-68

39 Franke AG, Bonertz C, Christmann M et al. Non-medical use of prescription stimulants and illicit use of stimulants for cognitive enhancement in pupils and students in Germany. Pharmacopsychiatry 2011; 43: 1-7

40 Franke AG, Konrad A, Lieb K et al. Psychostimulantien und Nicht-Stimulantien in der heutigen und zukünftigen ADHS-Therapie. Fortschr Neurol Psychiat 2011; 79: 1-11

41 Singh I, Kelleher KJ. Neuroenhancement in Young People: Proposal for Research, Policy, and Clinical Management. AJOB Neuroscience 2010 $1(1): 3-16$

42 Singh I, Kendall T, Taylor $C$ et al. Young people's experience of ADHD and stimulant medication: A qualitative study for the NIC guideline. Child Adol Ment Health 2010; 15 (4): 186-192

43 Singh I. A disorder of anger and aggression: Children's perspectives on attention deficit/hyperactivity disorder in the UK. Soc Scie \& Med 2011; 73 (6): 889-896

44 Minzenberg MJ, Carter CS. Modafinil: a review of neurochemical actions and effects on cognition. Neuropsychopharmacology 2008; 33 (7): $1477-1502$

45 Repantis D, Laisney 0 , Heuser I et al. Acetylcholinesterase inhibitors and memantine for neuroenhancement in healthy individuals: A systematic review. Pharmacol Res 2010; 61 (6): 473-481

46 Repantis D, Schlattmann P, Laisney $O$ et al. Antidepressants for neuroenhancement in healthy individuals: A systematic review. Poiesis \& Praxis 2009; 6 (3): 139-174

47 Slingsby $B$. The Prozac boom and its placebogenic counterpart - a culturally fashioned phenomenon. Med Sci Monit 2002; 8 (5): CR389CR393

48 DAK (Deutsche Angestellten-Krankenkasse). Gesundheitsreport 2009. Analyse der Arbeitsunfähigkeitsdaten. Schwerpunktthema Doping am Arbeitsplatz. Berlin: 2009, http://www.dak.de/content/filesopen/Ge sundheitsreport_2009.pdf
49 McCabe SE, Knight JR, Teter CJ et al. Non-medical use of prescriptions stimulants among US college students: prevalence and correlates from a national survey. Addiction 2005; 100 (1): 96-106

50 Franke A, Lieb K. Pharmakologisches Neuroenhancement und „Hirndoping": Chancen und Risiken. Bundesgesundheitsbl 2010; 53: 853-858

51 Maher B. Poll results: look who's doping. Nature 2008; 452: 674-675

52 Sahakian BJ, Morein-Zamir S. Professor's little helper. Nature 2007; 450: $1157-1159$

53 Schleim S, Walter H. Cognitive Enhancement - Fakten und Mythen. Nervenheilkunde 2007; 26: 83-87

54 Galert T, Bublitz C, Heuser I et al. Das optimierte Gehirn. Ein Memorandum zu Chancen und Risiken des Neuroenhancements. Gehirn \& Geist 2009; $11: 40-48$

55 Douglas T. Moral enhancement. J Appl Philos 2008; 25: 228 - 245

56 Harris J. Moral enhancement and freedom. Bioethics 2011; 25 (2): $102-111$

57 Persson I, Savulescu J. The perils of cognitive enhancement and the urgent imperative to enhance the moral character of humanity. J Appl Philos 2008; 25: 162 - 177

58 Fröding BEE. Cognitive enhancement, virtue ethics and the good life. Neuroethics 2011; 4: 223-234

59 Moynihan R, Doran E, Henry D. Disease mongering is now part of the global health debate. PLoS Med 2008; 5 (5): e106. DOI: 10.1371/journal.pmed.0050106

60 Public Library of Science. A collection of articles on disease mongering. PLoS Med 2008; 3 (4): e191. DOI: 10.1371/journal.pmed.0030191 http://collections.plos.org/plosmedicine/diseasemongering-2006.php

61 Davis K. Rethinking „normal”. Hast Cen Rep 2007; 37 (3): $44-47$

62 Metzinger $T$. The no-self-alternative (Chapter 11). In: Gallagher S (ed.) The Oxford Handbook of the Self Oxford: Oxford University Press 2011, 279-296

63 Metzinger T. Der Ego-Tunnel. Berlin: Bloomsbury 2011

64 Metzinger $T$. Why are identity-disorders interesting for philosophers? In: Schramme T, Thome J (eds.) Philosophy and Psychiatry Berlin: de Gruyter 2004, 311 - 325

65 Metzinger T. Being No One. The Self-Model Theory of Subjectivity. Cambridge: MIT Press 2003

66 Bolt I, Schermer M. Psychopharmaceutical enhancers: enhancing identity? Neuroethics 2008; 2: $103-111$

67 Olson ET. Personal identity. In: Zalta EN (ed.) The Stanford Encyclopedia of Philosophy.(Winter 2010 Edition) http://plato.stanford.edu/archives/win2010/entries/identity-personal/ [Epub only]

68 Shoemaker D. Personal identity and ethics. In: Zalta EN (ed.) The Stanford Encyclopedia of Philosophy.(Winter 2010 Edition) http://plato. stanford.edu/archives/fall2008/entries/identity-ethics/ [Epub only]

69 DeGrazia D. Human Identity and Bioethics. Cambridge: Cambridge University Press 2005

70 Galert T. Wie mag Neuro-Enhancement Personen verändern? In: Schöne-Seifert B, Talbot D, Opolka U, Ach JS (Hrsg) Neuro-Enhancement. Ethik vor neuen Herausforderungen Paderborn: mentis 2008, 159187

71 Gillett G. Subjectivity and Being Somebody: Human Identity and Neuroethics. Exeter: Imprint 2008

72 Merkel R, Boer R, Fegert J et al. Intervening in the brain - Changing Psyche and Society. Berlin: Springer 2007

73 Bublitz JC, Merkel R. Autonomy and authenticity of enhanced personality traits. Bioethics 2009; 23 (6): $360-374$

74 Califano J. "You've got drugs!" Prescription drug pushers on the internet. New York: Columbia University: The National Center on Addiction and Substance Abuse 2008, 1-15

75 Sententia $W$. Neuroethical considerations: Cognitive liberty and converging technologies for improving human cognition. Ann N Y Acad Sci 2004; 1013: $221-228$

76 Capps B. Libertarianism, legitimation, and the problems of regulating cognition-enhancing drugs. Neuroethics 2011; 4: 119-128

77 Nagel SK. Too much of a good thing? Enhancement and the burden of self-determination. Neuroethics 2010; 3: 109-119

78 Nagel SK. Ethics and the Neurosciences: Ethical and Social Consequences of Neuroscientific Progress. Paderborn: mentis 2010 